\title{
On abundance theorem for semi log canonical threefolds
}

\author{
By Osamu Fujino*) \\ Research Institute for Mathematical Sciences, Kyoto University, Kita-Shirakawa-Oiwake-cho, Sakyo-ku, Kyoto 606-8502 \\ (Communicated by Shigefumi MorI, M. J. A., June 15, 1999)
}

\begin{abstract}
Let $(X, \Delta)$ be a proper semi log canonical threefold with $K_{X}+\Delta$ nef. Then $K_{X}+\Delta$ is semi-ample.
\end{abstract}

Key word: Abundance Theorem.

1. Introduction. The main purpose of this paper is to announce the abundance theorem for semi $\log$ canonical threefolds (for the proof, see [3]). The abundance conjecture is a very important problem in the birational classification of algebraic varieties.

By our method we can reduce the abundance conjecture for semi log canonical pairs to the irreducible case and the finiteness of some groups. This shows that if the log Minimal Model Program (log MMP, for short), the log abundance conjecture, and the finiteness of B-pluricanonical representations (see Section 4) hold for $n$-folds, then the log abundance conjecture for semi $\log$ canonical $n$-folds is true almost automatically (see Theorem 5.10). But unfortunately the log MMP and the log abundance conjecture are only conjectures for $n$-folds with $n \geq 4$. So we prove

Theorem 1.1 (Abundance theorem for slc 3folds). Let $(X, \Delta)$ be a proper semi log canonical threefold with $K_{X}+\Delta$ nef. Then $K_{X}+\Delta$ is semiample.

This theorem is a generalization of the log abundance theorem for 3-folds proved by S. Keel, K. Matsuki and J. McKernan (see [12]). According to them, the $\log$ abundance theorem for threefolds is considered to be the first step towards a proof of the abundance conjecture in dimension four. We believe that the abundance theorem for semi log canonical threefolds is the second step.

\section{Notation.}

(1) The word scheme is used for schemes which are separated and of finite type over $\mathbf{C}$ and the word variety stands for a reduced and irreducible scheme. When we say a normal scheme, we mean the disjoint union of irre-

\footnotetext{
*) Research Fellow of the Japan Society for the Promotion of Science.
}

ducible normal schemes.

(2) We use the notation that $(X, \Delta)$ being Kawamata log terminal, divisorial log terminal, and log canonical (frequently abbreviated as klt, dlt, and lc), as in [15] (see also [20]). In the definition in [15, Section 2.3] $\Delta$ is not necessarily effective, but in this paper we assume $\Delta$ is an effective $\mathbf{Q}$-divisor.

(3) The log MMP means the log MMP for Qfactorial dlt pairs.

2. Definitions and preliminaries. In this section, we make some basic definitions.

Definition 2.1. Let $X$ be a reduced $S_{2}$ scheme. We assume that it is pure $n$-dimensional and normal crossing in codimension 1 . Let $\Delta$ be an effective Q-Weil divisor on $X$ (cf. [14, 16.2]) such that $K_{X}+\Delta$ is $\mathbf{Q}$-Cartier.

Let $X=\cup X_{i}$ be a decomposition into irreducible components, and $\mu: X^{\prime}:=\coprod X_{i}^{\prime} \rightarrow X=$ $\cup X_{i}$ the normalization. A Q-divisor $\Theta$ on $X^{\prime}$ is defined by $K_{X^{\prime}}+\Theta:=\mu^{*}\left(K_{X}+\Delta\right)$ and a $\mathbf{Q}$-divisor $\Theta_{i}$ on $X_{i}^{\prime}$ by $\Theta_{i}:=\left.\Theta\right|_{X_{i}^{\prime}}$.

We say that $(X, \Delta)$ is a semi log canonical $n$-fold (an slc $n$-fold, for short) if $\left(X_{i}^{\prime}, \Theta_{i}\right)$ is lc for every $i$.

We say that $(X, \Delta)$ is a semi divisorial log terminal $n$-fold (an sdlt $n$-fold, for short) if $X_{i}$ is normal, that is, $X_{i}^{\prime}$ is isomorphic to $X_{i}$, and $\left(X_{i}, \Theta_{i}\right)$ is dlt for every $i$.

The notion of semi log canonical pairs was first introduced in [16] for the problem of compactifying the moduli of surfaces. For the further development of this direction, we recommend the readers to see [7].

\section{Remark 2.2.}

(1) The slc in Definition 2.1 is equivalent to the slc in [14, Chapter 12] (see [13, 4.2]).

(2) If $(X, \Delta)$ is an slc $n$-fold, then $X$ is seminor- 
mal (see [14, 12.2.1.(8)] and [1, Remark 4.7]).

(3) If $(X, \Delta)$ is a dlt $n$-fold, then $(\llcorner\Delta\lrcorner, \operatorname{Diff}(\Delta-$ $\llcorner\Delta\lrcorner))$ is an sdlt $(n-1)$-fold (see $[14,17.5]$ and $[15,5.52])$.

(4) Let $(X, \Delta)$ be lc. Then $(\llcorner\Delta\lrcorner, \operatorname{Diff}(\Delta-\llcorner\Delta\lrcorner))$ is not necessarily slc (see [14, 17.5.2 Example]). Note that $[14,(16.9 .1)]$ is not true.

Definition 2.3. Let pairs $(X, \Delta)=$ $\coprod_{i=1}^{n}\left(X_{i}, \Delta_{i}\right)$ and $\left(X^{\prime}, \Delta^{\prime}\right)=\coprod_{i=1}^{n}\left(X_{i}^{\prime}, \Delta_{i}^{\prime}\right)$ be normal schemes with $\mathbf{Q}$-divisors such that $K_{X}+\Delta$ and $K_{X^{\prime}}+\Delta^{\prime}$ are Q-Cartier Q-divisors.

We say that a map $f:(X, \Delta) \rightarrow\left(X^{\prime}, \Delta^{\prime}\right)$ is a B-birational map (resp. morphism) if $f: X \rightarrow$ $X^{\prime}$ is a proper birational map (resp. morphism) and there exists a common resolution $\alpha: T \rightarrow X, \beta$ : $T \rightarrow X^{\prime}$ of $f: X \rightarrow X^{\prime}$ such that $\alpha^{*}\left(K_{X}+\Delta\right)=$ $\beta^{*}\left(K_{X^{\prime}}+\Delta^{\prime}\right)$. That is, there exists a permutation $\sigma$ such that $f_{i}: X_{i} \rightarrow X_{\sigma(i)}^{\prime}$ is a proper birational map (resp. morphism) and there exists a common resolution $\alpha_{i}: T_{i} \rightarrow X_{i}, \beta_{i}: T_{i} \rightarrow X_{\sigma(i)}^{\prime}$ of $f_{i}$ such that $\alpha_{i}^{*}\left(K_{X_{i}}+\Delta_{i}\right)=\beta_{i}^{*}\left(K_{X_{\sigma(i)}^{\prime}}+\Delta_{\sigma(i)}^{\prime}\right)$ on $T_{i}$ for every $i$. The last condition means that if we write

$$
\begin{aligned}
& K_{T_{i}}=\alpha_{i}^{*}\left(K_{X_{i}}+\Delta_{i}\right)+F, \\
& K_{T_{i}}=\beta_{i}^{*}\left(K_{X_{\sigma(i)}^{\prime}}+\Delta_{\sigma(i)}^{\prime}\right)+E,
\end{aligned}
$$

then $F=E$.

If there exists a B-birational map from $(X, \Delta)$ to $\left(X^{\prime}, \Delta^{\prime}\right)$, we say that $(X, \Delta)$ is B-birational equivalent to $\left(X^{\prime}, \Delta^{\prime}\right)$. Here the symbol $B$ is the initial of boundary.

3. Reduced boundaries of dlt pairs. The following is a reformulation of [14, 12.3.2], which fits better in our arguments. It plays essential roles in the proof of Proposition 5.3, which is a key step of the proof of the main theorem.

Proposition 3.1 (cf. [19, 6.9], [14, 12.3.2]). Let $(X, \Theta)$ be a Q-factorial dlt $n$-fold with $n \leq 3$. Let $f: X \rightarrow R$ be a proper surjective morphism onto a normal variety $R$ with connected fibers. Assume that $K_{X}+\Theta$ is numerically $f$-trivial. Then one of the following holds:

(0) $\operatorname{dim} R=0$.

(0.1) $\llcorner\Theta\lrcorner$ is connected.

(0.2) $\llcorner\Theta\lrcorner$ has two connected components $\Delta_{1}$ and $\Delta_{2}$ and there exists a rational map $v: X \rightarrow$ $(V, P)$ onto a Q-factorial lc $(n-1)$-fold $(V, P)$ with general fiber $\mathbf{P}^{1}$. Furthermore there exists an irreducible component $D_{i}^{\prime} \subset$ $\Delta_{i}$ such that $\left.v\right|_{D_{i}^{\prime}}:\left(D_{i}^{\prime}, \operatorname{Diff}\left(\Theta-D_{i}^{\prime}\right)\right)-\rightarrow$
$(V, P)$ is a B-birational map for $i=1,2$.

(1) $\operatorname{dim} R \geq 1$.

(1.1) $\llcorner\Theta\lrcorner \cap f^{-1}(r)$ is connected for every $r \in R$.

(1.2) The number of connected components of $\llcorner\Theta\lrcorner \cap f^{-1}(r)$ is at most two for every $r \in R$. There exists a rational map $v: X \rightarrow(V, P)$ onto a $\mathbf{Q}$-factorial lc $(n-1)$-fold $(V, P)$ with general fiber $\mathbf{P}^{1}$. The horizontal part $\Theta^{h}$ of $\llcorner\Theta\lrcorner$ is one of the following:

(i) $\Theta^{h}=D_{1}^{\prime}$ which is irreducible and the mapping degree $\operatorname{deg}\left[D_{1}^{\prime}: V\right]=2$ and there is a B-birational involution on $\left(D_{1}^{\prime}, \operatorname{Diff}\left(\Theta-D_{1}^{\prime}\right)\right)$ over $V$.

(ii) $\Theta^{h}=D_{1}^{\prime}+D_{2}^{\prime}$ such that $D_{i}^{\prime}$ is irreducible and $\left.v\right|_{D_{i}^{\prime}}:\left(D_{i}^{\prime}, \operatorname{Diff}(\Theta-\right.$ $\left.\left.D_{i}^{\prime}\right)\right) \rightarrow(V, P)$ is a B-birational map for $i=1,2$.

\section{Remark 3.2.}

(1) In Proposition 3.1 the assumption $K_{X}+$ $\Theta \equiv{ }_{f} 0$ is equivalent to $K_{X}+\Theta \sim_{\mathbf{Q}, f} 0$. It is because the relative log abundance theorem holds when $\operatorname{dim} X \leq 3$.

(2) If the $\log$ MMP holds for $n$-folds, then Proposition 3.1 is also true for $n$-folds.

4. Finiteness of B-pluricanonical representations. We consider the birational automorphism groups of pairs.

Definition 4.1. Let $(X, \Delta)$ be a pair of a normal scheme and a $\mathbf{Q}$-divisor such that $K_{X}+\Delta$ is Q-Cartier. We define

$\operatorname{Bir}(X, \Delta):=\{\sigma: X \rightarrow X \mid \sigma$ is B-birational $\}$,

$\operatorname{Aut}(X, \Delta):=\left\{\begin{array}{l|l}\sigma: X \rightarrow X & \begin{array}{l}\text { The map } \sigma \text { is an } \\ \text { automorphism and } \\ \sigma^{*} \Delta=\Delta\end{array}\end{array}\right\}$.

Since $\operatorname{Bir}(X, \Delta)$ acts on $H^{0}\left(X, \mathcal{O}_{X}\left(m\left(K_{X}+\Delta\right)\right)\right)$ for every integer $m$ such that $m\left(K_{X}+\Delta\right)$ is a Cartier divisor, we can define B-pluricanonical representation $\rho_{m}: \operatorname{Bir}(X, \Delta) \rightarrow$ Aut $H^{0}\left(X, m\left(K_{X}+\Delta\right)\right)$.

The following conjecture plays an important role when we reduce the problem to the irreducible case.

Conjecture 4.2 (Finiteness of B-pluricanonical representations). Let $(X, \Delta)$ be an n-dimensional (not necessarily connected) proper lc pair. Assume that $K_{X}+\Delta$ is nef. Then there exists a positive integer $m_{0}$ such that $\rho_{m_{1} m_{0}}(\operatorname{Bir}(X, \Delta))$ is finite for every $m_{1} \in \mathbf{N}$.

For Conjecture 4.2 , it is obviously sufficient to prove it under the assumption that $X$ is irreducible. 
Theorem 4.3 (cf. $[14,12.2 .11])$. Let $(C, \Delta)$ be a proper lc curve. Then there exists a positive integer $m_{0}$ such that $\rho_{m_{1} m_{0}}(\operatorname{Aut}(C, \Delta))$ is finite for every $m_{1} \in \mathbf{N}$

Theorem 4.4. Let $(S, \Delta)$ be a proper klt surface. Then there exists a positive integer $m_{0}$ such that $\rho_{m_{1} m_{0}}(\operatorname{Bir}(S, \Delta))$ is finite for every $m_{1} \in \mathbf{N}$.

Theorem 4.5. Let $(S, \Delta)$ be a proper lc surface. Assume that $K_{S}+\Delta$ is nef. Then there is a positive integer $m_{0}$ such that $\rho_{m_{1} m_{0}}(\operatorname{Bir}(S, \Delta))$ is finite for every $m_{1} \in \mathbf{N}$.

5. The abundance theorem for slc pairs. We introduce the notion of preadmissible and admissible sections for the inductive proof of the abundance conjecture for slc $n$-folds.

Definition 5.1. Let $(X, \Delta)$ be a proper sdlt $n$-fold and $m$ a sufficiently large and divisible integer. We define admissible and preadmissible sections inductively on dimension.

(1) $s \in H^{0}\left(X, \mathcal{O}_{X}\left(m\left(K_{X}+\Delta\right)\right)\right)$ is preadmissible if the restriction $\left.s\right|_{\left(\amalg_{i}\left\llcorner\Theta_{i}\right\lrcorner\right)}$ is admissible in $H^{0}\left(\coprod_{i}\left\llcorner\Theta_{i}\right\lrcorner, \mathcal{O}_{\left(\amalg\left\llcorner\Theta_{i}\right\lrcorner\right)}\right)\left(m\left(K_{X^{\prime}}+\right.\right.$ $\left.\left.\Theta)\left.\right|_{\left(\amalg\left\llcorner\Theta_{i}\right\lrcorner\right)}\right)\right)$.

(2) $s \in H^{0}\left(X, \mathcal{O}_{X}\left(m\left(K_{X}+\Delta\right)\right)\right)$ is admissible if $s$ is preadmissible and $g^{*}\left(\left.s\right|_{X_{j}}\right)=\left.s\right|_{X_{i}}$ for every B-birational map $g:\left(X_{i}, \Theta_{i}\right) \rightarrow\left(X_{j}, \Theta_{j}\right)$ for every $i, j$.

Note that if $s \in H^{0}\left(X, \mathcal{O}_{X}\left(m\left(K_{X}+\Delta\right)\right)\right)$ is admissible, then $\left.s\right|_{X_{i}}$ is $\operatorname{Bir}\left(X_{i}, \Theta_{i}\right)$-invariant for every $i$.

We define linear subspaces of $H^{0}\left(X, \mathcal{O}_{X}\left(m\left(K_{X}+\Delta\right)\right)\right)$ as follows;

$\operatorname{PA}\left(X, m\left(K_{X}+\Delta\right)\right):=\{s$ is preadmissible $\}$,

and

$$
\mathrm{A}\left(X, m\left(K_{X}+\Delta\right)\right):=\{s \text { is admissible }\} .
$$

When $\operatorname{dim} X=1$, the preadmissible section is a slight generalization of the normalized section (see $[14,12.2 .9])$. But in the higher dimensional case, the admissible and preadmissible sections behave much better in the inductive proof of the abundance conjecture for slc $n$-folds.

Lemma 5.2. Let $(X, \Delta)$ be a proper Qfactorial dlt $n$-fold, and $K_{X}+\Delta$ nef and $S=\llcorner\Delta\lrcorner \neq$ 0 . Assume that $f=\Phi_{\left|k\left(K_{X}+\Delta\right)\right|}: X \rightarrow R$ is a proper surjective morphism onto a normal variety $R$ with connected fibers for a sufficiently large and divisible integer $k$ and $f(\llcorner\Delta\lrcorner) \subsetneq R$. If there exist sections $\left\{s_{i}\right\}_{i=1}^{p} \subset H^{0}\left(S, \mathcal{O}_{S}\left(\left.m\left(K_{X}+\Delta\right)\right|_{S}\right)\right)$ without common zeros, then there exist sections $\left\{u_{i}\right\}_{i=1}^{l} \subset$ $H^{0}\left(X, \mathcal{O}_{X}\left(r m\left(K_{X}+\Delta\right)\right)\right)$ for some integer $r$ such that

(1) $\left.u_{i}\right|_{S}= \begin{cases}s_{i}^{r} & \text { for } 1 \leq i \leq p \\ 0 & \text { for } p<i \leq l\end{cases}$

(2) $\left\{u_{i}\right\}_{i=1}^{l}$ have no common zeros.

The next proposition is the main part of the proof of the abundance theorem for slc $n$-folds, which is proved by Proposition 3.1 and Lemma 5.2.

Proposition 5.3. Let $(X, \Delta)$ be a pure $n$ dimensional (not necessarily connected) proper $\mathbf{Q}$ factorial dlt pair with $n \leq 3$. Let $m$ be a sufficiently large and divisible integer. Assume that

(1) $K_{X}+\Delta$ is nef,

(2) $\mathcal{O}_{\llcorner\Delta\lrcorner}\left(m\left(K_{\llcorner\Delta\lrcorner}+\operatorname{Diff}(\Delta-\llcorner\Delta\lrcorner)\right)\right)$ is generated by $\mathrm{A}\left(\llcorner\Delta\lrcorner,\left.m\left(K_{X}+\Delta\right)\right|_{\llcorner\Delta\lrcorner}\right)$.

Then

$\mathrm{PA}\left(X, m\left(K_{X}+\Delta\right)\right) \rightarrow \mathrm{A}\left(\llcorner\Delta\lrcorner,\left.m\left(K_{X}+\Delta\right)\right|_{\llcorner\Delta\lrcorner}\right)$

is surjective, and $\mathcal{O}_{X}\left(m\left(K_{X}+\Delta\right)\right)$ is generated by $\operatorname{PA}\left(X, m\left(K_{X}+\Delta\right)\right)$.

Remark 5.4. In Proposition 5.3 the assumption $n \leq 3$ is used for the $\log$ MMP and the log abundance theorem which are used in Proposition 3.1 too. lemma.

By Lemma 5.7 and Section 4, we get the next

Lemma 5.5. In Proposition 5.3, if $\operatorname{dim} X \leq 2$, then we can replace $\mathrm{PA}\left(X, m\left(K_{X}+\Delta\right)\right)$ by the linear subspace $\mathrm{A}\left(X, m\left(K_{X}+\Delta\right)\right)$.

In order to prove Lemma 5.7 we need the next definition.

Definition 5.6. Assume that $X$ is nonsingular and $\operatorname{Supp} \Delta$ is a simple normal crossing divisor and $\Delta=\sum_{i} d_{i} \Delta_{i}$ is a $\mathbf{Q}$-divisor such that $d_{i} \leq 1$ for every $i$. In this case we say that $(X, \Delta)$ is B-smooth.

Let $(X, \Delta)$ be dlt or B-smooth. A subvariety $W$ of $X$ is said to be a center of log canonical singularities if there is a proper birational morphism from a normal variety $\mu: Y \rightarrow X$ and a prime divisor $E$ on $Y$ with the discrepancy $a(E, X, \Delta)=-1$ such that $\mu(E)=W$ (cf. [10, Definition 1.3]).

Let $(X, \Delta)$ be dlt or B-smooth. We write $\Delta=$ $\sum_{i} d_{i} \Delta_{i}$ such that $\Delta_{i}$ are distinct prime divisors. Then the B-part of $\Delta$ is defined by $\Delta^{B}:=\sum_{d_{i}=1} \Delta_{i}$.

If $(X, \Delta)$ is dlt or B-smooth, then a center of $\log$ canonical singularities is an irreducible component of an intersection of some B-part divisors. (see the Divisorial Log Terminal Theorem of [20] and [15, Sec- 
tion 2.3].) When we consider a center of log canonical singularities $W$, we always consider the pair $(W, \Xi)$ such that $K_{W}+\Xi=\left.\left(K_{X}+\Delta\right)\right|_{W}$, where $\Xi$ is defined by using the adjunction repeatedly. Note that if $(X, \Delta)$ is dlt (resp. B-smooth), then $(W, \Xi)$ is dlt (resp. B-smooth) by the adjunction.

If $(X, \Delta)$ is dlt pair or B-smooth and $W$ is a center of $\log$ canonical singularities, then we write $W \Subset X$.

Lemma 5.7. Let $(X, \Delta)$ be a pure n-dimensional (not necessarily connected) proper dlt pair with $K_{X}+\Delta$ nef and let $m$ be a sufficiently large and divisible integer. We write $G=\rho_{m}(\operatorname{Bir}(X, \Delta))$. If $s \in \mathrm{PA}\left(X, m\left(K_{X}+\Delta\right)\right)$, then $g^{*} s \in \mathrm{PA}\left(X, m\left(K_{X}+\right.\right.$ $\Delta))$ for every $g \in G$ and $\left.g^{*} s\right|_{\llcorner\Delta\lrcorner}=\left.s\right|_{\llcorner\Delta\lrcorner}$.

In particular if $|G|$ is finite, then

$$
\begin{gathered}
\sum_{g \in G} g^{*} s \in \mathrm{A}\left(X, m\left(K_{X}+\Delta\right)\right), \\
\prod_{g \in G} g^{*} s \in \mathrm{A}\left(S, m|G|\left(K_{X}+\Delta\right)\right),
\end{gathered}
$$

and

$$
\left.\prod_{g \in G} g^{*} s\right|_{\llcorner\Delta\lrcorner}=\left(\left.s\right|_{\llcorner\Delta\lrcorner}\right)^{|G|} .
$$

For the proof of this lemma we use the next two claims and Szabó's resolution lemma, which is a powerful tool of treating dlt pairs (see [4]).

Claim $\left(\mathbf{A}_{n}\right)$. Let $(T, \Theta)$ and $(S, \Xi)$ be $n$ dimensional B-smooth pairs and $h: S \rightarrow T$ a Bbirational morphism. If $W \Subset T$, then there is a $W^{\prime} \Subset S$ such that $W^{\prime} \rightarrow W$ is a B-birational morphism.

Claim $\left(\mathbf{B}_{\boldsymbol{n}}\right)$. Let $(T, \Theta)$ and $(S, \Xi)$ be $n$ dimensional B-smooth pairs and $h: S \rightarrow T$ a Bbirational morphism. Assume that $W \Subset S$. If $W \rightarrow h(W)$ is not B-birational, then there is a $W^{\prime} \Subset W$ such that $W^{\prime} \rightarrow h(W)$ is a B-birational morphism and that the inclusion $W^{\prime} \rightarrow W$ induces the isomorphism $H^{0}\left(W, \mathcal{O}_{W}\left(\left.m\left(K_{S}+\Xi\right)\right|_{W}\right)\right) \simeq$ $H^{0}\left(W^{\prime}, \mathcal{O}_{W^{\prime}}\left(\left.m\left(K_{S}+\Xi\right)\right|_{W^{\prime}}\right)\right)$.

By Proposition 5.3, we have the following results.

Corollary 5.8 (cf. $[14,12.1 .1,8.5])$. Let $(X, \Delta)$ be a proper slc $n$-fold such that $K_{X}+\Delta$ is nef, where $n \leq 2$. Then $\left|m\left(K_{X}+\Delta\right)\right|$ is free for a sufficiently large and divisible integer $m$. In particular, if $(X, \Delta)$ is proper sdlt, then $\mathcal{O}_{X}\left(m\left(K_{X}+\Delta\right)\right)$ is generated by $\mathrm{A}\left(X, m\left(K_{X}+\Delta\right)\right)$.
The next corollary is the main theorem of this paper (see Theorem 1.1).

Corollary 5.9. Let $(X, \Delta)$ be a proper slc threefold with $K_{X}+\Delta$ nef. Then $K_{X}+\Delta$ is semiample.

We can reformulate Corollaries 5.8 and 5.9 in the following form if we list all the necessary results (e.g. $\log$ MMP) as the assumption.

Theorem 5.10. Assume the log MMP for dimension $n$. If the abundance conjecture holds for $l c$ $n$-folds and if the finiteness of B-pluricanonical representations (see Conjecture 4.2) is true for dimension $n$, then the abundance conjecture is true for sdlt and slc $n$-folds, that is, Corollary 5.8 holds in dimension $n$.

Acknowledgements. I would like to express my sincere gratitude to Prof. Shigefumi Mori, M.J.A. for giving me advice. I am grateful to Profs. Noboru Nakayama and Yoichi Miyaoka for giving me many useful comments.

\section{References}

[ 1 ] F. Ambro: The locus of log canonical singularities. (1998) (preprint).

[ 2 ] A. Beauville: Complex Algebraic Surfaces, 2nd ed. London Math. Soc. Stud. Texts, 34 (1996).

[ 3 ] O. Fujino: Abundance theorem for semi log canonical threefolds. RIMS-1213 (1998) (preprint).

[ 4 ] O. Fujino: Base point free theorem of Reid-Fukuda type (1999) (preprint).

[ 5 ] T. Fujita: Fractionally logarithmic canonical rings of surfaces. J. Fac. Sci. Univ. Tokyo. 30, 685-696 (1984).

[ 6 ] S. Iitaka: Algebraic Geometry, An Introduction to Birational Geometry of Algebraic Varieties. Grad. Texts in Math., 76, Springer (1981).

[ 7 ] K. Karu: Minimal models and boundedness of stable varieties. preliminary version (1998) (preprint).

[ 8 ] Y. Kawamata: Pluricanonical systems on minimal algebraic varieties. Inv. Math., 79, 567-588 (1985).

[ 9 ] Y. Kawamata: Abundance theorem for minimal threefolds. Inv. Math., 108, 229-246 (1992).

[10] Y. Kawamata: On Fujita's freeness conjecture for 3-folds and 4-folds. Math. Ann., 308, 491-505 (1997).

[11] Y. Kawamata, K. Matsuda, and K. Matsuki: Introduction to the Minimal Model Problem, in Algebraic Geometry, Sendai 1985. Adv. Stud. Pure Math., 10, Kinokuniya and North-Holland, 283360 (1987).

[12] S. Keel, K. Matsuki, and J. McKernan: Log abun- 
dance theorem for threefolds. Duke Math. J., 75, 99-119 (1994).

[13] J. Kollár: Projectivity of complete moduli. J. Differential Geom., 32, 235-268 (1990).

[14] J. Kollár et al:: Flips and Abundance for Algebraic Threefolds. Astérisque, 211, Soc. Math. France (1992).

[15] J. Kollár and S. Mori: Birational geometry of algebraic varieties. Cambridge Tracts in Math., 134 (1998).

[16] J. Kollár and N. Shepherd-Barron: Threefolds and deformations of surface singularities. Invent. Math., 91, 299-338 (1988).

[17] D. Mumford: Abelian Varieties. Oxford Univ.
Press, pp. 1-242 (1970).

[18] F. Sakai: Kodaira dimensions of complements of divisors, in Complex Analysis and Algebraic Geometry. Iwanami and Cambridge Univ. Press, pp. 239-257 (1977).

[19] V. V. Shokurov: 3-fold log flips. Izv. Ross. Akad. Nauk Ser. Mat., 56, 105-203 (1992); Russian Acad. Sci. Izv. Math. 40, 95-202 (1993) (translated in English).

[20] E. Szabó: Divisorial log terminal singularities. J. Math. Sci. Univ. Tokyo, 1, 631-639 (1995).

[21] K. Ueno: Classification Theory of Algebraic Varieties and Compact Complex Spaces. Springer Lecture Notes, vol. 439, pp. 1-278 (1975). 